\title{
La estructura práctica de la acción educativa. Esbozo de un campo de investigación
}

\author{
FERNANDO BÁRCENA ORBE \\ Universidad Complutense de Madrid
}

\begin{abstract}
SUMMARY.-In this paper, the author attempts to show the essentialy contested character of the concept of education. Frecuently, education has been presented as a human practical action. However, in many contexts this thesis has been employed to elaborate a reflective and ethical perspective of educational profesion, as an alternative approach to a technological one, or, to the contrary, to justify the primacy of technological rationality on moral rationality in the educational process. In order to achieve a holistic perspective of education, both claims must be avoided. Concretely, the author propose to make up the tradition of practical philosophy, in which technical and ethical dimension of human action shaping a practical structure particularly linked with forms of practical cognition. In addition to this, the paper attempts to check some of the most important contributions in this field of educational research.
\end{abstract}

\section{INTRODUCCIÓN}

Está bastante extendida la convicción en el ámbito de la investigación pedagógica de que toda acción educativa es, sustancialmente, un modo práctico de intervención. Considerada en términos de «acción» la actuación educativa, pedagógicamente planificada, es una acción práctica que posee una lógica y discurso específico, distinto, aunque no contrapuesto, a la lógica del discurso teórico. La diferencia esencial entre ambas modalidades de discurso se encuentra en que mientras el primero tiende a la realización de procesos de deliberación, construcción de juicios prácticos y toma de decisiones, el segundo busca la producción de nuevos saberes y la elaboración fundada de proposiciones universalizables.

La atención especial que numerosos teóricos y filósofos de la educación han prestado al análisis de la cognición práctica en la acción educativa y a la investigación de los procesos de pensamiento, juicio y toma de decisiones pedagógicas, sin duda ha contribuido a delimitar un campo de investigación verdaderamente interdisciplinar, que aún puede verse potenciado desde la perspectiva de las denominadas «ciencias cognitivas». En este trabajo el acento principal se pondrá en la perspectiva de la filosofía de la educación -entendida como filosofía práctica de la acción educativa- y mi propósito principal será intentar esbozar los delineamientos, posibles líneas de trabajo y temas fundamentales de dicho campo de investigación. 
Concretamente esta revisión se sitúa en el punto de confluencia de dos debates pedagógicos principales, los cuales, en los últimos años, han generado una extensa producción bibliográfica cuyo núcleo fundamental está contribuyendo a la reconsideración de la acción educativa como una práctica reflexiva.

El primer debate -de índole epistemológico- se refiere a la consideración de la educación como disciplina científica, susceptible de generar un conocimiento pedagógico científicamente autónomo y capaz de predecir y controlar regularidades entre diferentes acontecimientos educativos (Belth, 1971; Touriñán, 1987). El segundo debate, relacionado con el análisis de los procesos de profesionalización de las prácticas sociales, se refiere básicamente a la discusión de los criterios que deben definir la condición profesional de las actividades educativas y de los principales modelos de racionalidad en que se asienta la pretensión profesionalizadora en el ámbito de la educación (Carr, 1989; Goodland, Soder y Sirotnik, 1990; Kultgen, 1988; Novoa, 1987; Schön, 1983; 1987).

Ambos tipos de debates han promovido distintas clases de expectativas en nuestro campo. Algunas de ellas conectan directamente con planteamientos innovadores sobre la cuestión de la relación entre pensamiento pedagógico y acción educativa; otras han pretendido promover la reconsideración del papel que las diversas tecnologías pueden llegar a ejercer en el ámbito educativo -procurando favorecer con ello un cambio de perspectiva ante la tecnología educativa misma, la cual ha pasado a concebirse hoy como tecnología eminentemente procesual, cognitiva e intelectual- $y$, finalmente, un último conjunto de expectativas han revitalizado el interés por el estudio del carácter práctico de la actividad educativa, de sus principales dimensiones críticas y principios explicativos (Carr, 1987; Daniels, 1975; Damiano, 1988; Elliot, 1990; Groundy, 1991; Harnett y Naish, 1978; Hohan, 1990; Jackson, 1986; Knight y Smith, 1989; Kratochwill, 1987; Langford, 1989; Manen, 1991; Macdonald, 1992; Mackenzie, 1991; Pearson, 1989; Reid, 1978; Sockett, 1989).

Este artículo, que pretende ser una contribución teórica a esta última cuestión y sentar algunas bases para la formulación de una teoría de la educación como práctica reflexiva, reconoce su deuda filosófica con varias tradiciones de pensamiento. En primer lugar, con la tradición de pensamiento aristotélico sobre la racionalidad práctica, la cual modernamente se ha visto enriquecida, de un lado, por Gadamer -al haber resaltado la dimensión comprensiva e interpretativa del concepto de conocimiento práctico aristotélico (Gadamer, 1967, 1977 y 1981) - y, por otro, por Habermas, quien a través de su teoría crítica y de la acción comunicativa añade a las nociones de los anteriores autores el concepto de «competencia comunicativa» y una fuerte dimensión intersubjetiva, volviendo con ello más comunicable el saber que el conocimiento práctico produce (Habermas, 1987 y 1989). La segunda fuente de inspiración de este estudio se encuentra en Dewey -tanto en su teoría de la investigación como en sus formulaciones sobre la incertidumbre y el pensamiento reflexivo (Dewey, 1950, 952, 1966, 1989)- y en Schön y Argyris, quienes, tanto conjunta como separadamente, han contribuido a la reorientación de la enseñanza y el aprendizaje de las profesiones y a la reformulación del pensamiento reflexivo y la investigación acción (Schön, 1983, 1987, 1992; Argyris y otros, 1987; Argyris y Schön, 1974).

Pero este estudio debe mucho también a una tercera fuente teórica, a saber: a las teorías psicológicas constructivistas y estructurantes -de cuyo éxito en la aplicación pedagógica de sus constructos teóricos dan cuenta precisamente las teorías del aprendizaje 
significativo y del aprendizaje por reestructuración. Al menos dos de sus más evidentes consecuencias o implicaciones pedagógicas se tienen en cuenta en este estudio, las cuales confirman el carácter que la práctica educativa tiene de acción. En primer lugar, que la relación entre los procesos de enseñanza y aprendizaje no define una relación de causa-efecto, sino que aquélla está destinada a promover una actividad mental autoestructurante -unos determinados principios de acción en el alumno, el cual no mantiene con el objeto de conocimiento una relación meramente individual-, la cual es fuente del aprendizaje significativo. Aquí, lo que se ha dado en llamar «ayuda pedagógica», la intervención del educador, es fundamental. Y precisamente de esta ayuda pedagógica se pretende inferir en este estudio su carácter fuertemente reflexivo. En cualquier caso esta primera idea muestra que la educación, como actividad del alumno y del docente, se constituye como acción. La segunda implicación tiene que ver con la actuación docente y el modo en que utiliza y aplica el conocimiento en su actividad. También en este contexto debe darse necesariamente una mediación reflexiva. El saber se articula en una estructura cognitiva de mediación reflexiva configurándose como plan interior de acción, siendo su fin la conformación de una situación pedagógica en situación educativa.

Aunque estas conclusiones son por igual sumamente pertinentes a la hora de explorar la estructura práctica de la acción educativa, y la génesis del aprendizaje en el educando, o en su caso, del saber pedagógico en el educador, interesa, sobre todo, subrayar la importancia de la segunda tesis formulada, ya que en ella se sitúa -como he mostrado en otro lugar (Bárcena, 1993)- la raíz de la naturaleza reflexiva del acto pedagógico. Teniendo en cuenta, por tanto, la naturaleza y objetivo de este artículo él mismo estará dividido en cuatro secciones fundamentales.

En la primera parte apuntaré algunas razones por las cuales es útil realizar un mejor uso de la confrontación crítica entre tradiciones de pensamiento e investigación educativa rivales para esclarecer el sentido de la educación como acción práctica, dada la esencial contestabilidad que recorre, desde el punto de vista lógico, al concepto mismo de educación. Además, insistiré en la idea de que la investigación educativa, dado el carácter de práctica que la educación tiene en sí misma, puede obtener sus mayores cotas de desarrollo cuando se adopta un sentido fuerte de comunidad de investigación y aprendizaje, dentro de la cual los investigadores reconocen su deuda intelectual con una tradición de pensamiento que se vuelve racional, y en buen orden, en la medida que la abren a la confrontación crítica con otras tradiciones rivales. Aunque en el fondo exista siempre un núcleo de intraducibilidad entre las diversas tradiciones, lo cierto es que la discrepancia en ciertos aspectos, entre dos tradiciones rivales, muestra la medida en que sus respectivos lenguajes han sido, previsiblemente, asimilados y entendidos por los otros. Desde este punto de vista, y aunque sólo sea por esto, el fruto de tal confrontación siempre será la consecución de una mayor racionalidad en nuestras propias discrepancias.

La segunda parte estará dedicada a encuadrar el actual debate sobre la naturaleza de la acción educativa dentro de un marco explicativo de mayor alcance, el cual está presidido por el interés de revitalizar, justamente, la tradición del pensamiento e investigación aristotélica, tradición para la cual las relaciones entre pensamiento y acción adquieren un matiz distinto y realmente innovador.

En la tercera parte se revisarán brevemente algunas de las más significativas contribuciones al estudio de uno de los conceptos particularmente implicados en el análisis de 
la intervención educativa como acción, a saber: la naturaleza e implicaciones de la cognición práctica en la acción pedagógica.

La última parte del trabajo intentará destacar aspectos de interés y todavía necesitados de mayor atención y análisis, relacionados con la estructura o configuración de la educación como actividad práctica.

\section{EL CONOCIMIENTO DE LA EDUCACIÓN Y LA RACIONALIDAD DE LA TRADICIÓN}

Cualquier intento que pretenda discutir filosóficamente, y en profundidad, la naturaleza de la acción educativa exige una explícita referencia al modo en que ésta se constituye prácticamente, esto es, a la forma, o formas, en que la actualización educativa se configura como una compleja práctica humana. Cuando se intenta una tarea de estas características, lo normal es recurrir a la distinción aristotélica entre praxis -o acción- y poieseis $-\mathrm{o}$ actividad-, con el objeto de ofrecer una visión completa, y lo menos reduccionista posible, de la educación como actuación humana (Bárcena, 1989).

Ahora bien, cuando se procede de este modo se hace algo más que recurrir a un sistema de pensamiento -en este caso el aristotélico- antiguo. En el fondo, lo que se hace es recurrir a una tradición de pensamiento, investigación y práctica. Y al recurrir a ella se hace la tradición misma un método de investigación y de conocimiento.

Muy particularmente, desde la tradición aristotélica se ponen en evidencia dos fuertes afirmaciones, las cuales comparten un presupuesto común, que deseo comenzar a discutir en este artículo, cuyo propósito es revisar el importante número de aportaciones que actualmente están repensando el carácter práctico de la actuación educativa. La primera afirmación es que la educación es un concepto esencialmente contestable y polémico, una noción cuyo esclarecimiento depende de la confrontación crítica de diversas tradiciones pedagógicas. Y la segunda que la educación es, por encima de todo, una actividad moral que no entra en contradicción, bajo determinadas condiciones, con planteamientos tecnológicos (Bárcena, Gil, Jover, 1992 y 1993).

El presupuesto común que comparten estas afirmaciones es el papel fundamental que las diversas tradiciones intelectuales sobre educación están destinadas a cumplir en la conformación moral y pedagógica del acto educativo. Aunque el acto de autodefinición y autoposesión del hombre, como sujeto ético, es un acto inexcusablemente personal, sin embargo la situación humana, en tanto que tal, es significativamente una co-situación, esto es, una situación de convivencia, dado que la situación de los demás afecta directamente a mi acto personal de autodefinición y autoposesión. De acuerdo con esta consideración antropológica, se explica que la vida humana tenga una fuerte dimensión social e histórica. Y la acción que contribuye, por encima de todas las posibles, a la conformación humanizadora del hombre es, justamente, la educación. De este modo, puede señalarse, por un lado, que en tanto que humanos no partimos de cero, sino justamente de un legado y una tradición que es necesario incorporar significativamente y con la cual confrontar para lograr un sentido unitario de la vida humana. Y precisamente este hecho, el tener que realizar un proyecto coherente y unitario de vida humana es lo que no se puede hacer separadamente de la comunidad y de una tradición de la cual somos sus genuinos herederos. Por la misma razón, en segundo término, la tradición juega un papel fundamental en la explicación de la acción educativa en tanto que acción humana y práctica social. 
La importancia del concepto de tradición es, por tanto, doble. Es importante para dar sentido al concepto de educación, el cual alcanza sus más amplias posibilidades de desarrollo lógico mediante la confrontación de las diversas tradiciones culturales e intelectuales de pensamiento pedagógico. Simultáneamente, la tradición se nos presenta como una forma de conocimiento sustancialmente práctico. En efecto, como veremos en seguida, toda tradición incorpora una cierta teoría del conocimiento. Este va a ser el primer asunto que voy a tratar.

La idea que he planteado en primer término es parcialmente aporética. Como concepto, la educación es definible y delimitable. Pero como concepto esencialmente contestable, la definición del término que nos ocupa no tiene fácil cabida en una estipulación teórica acabada y concreta. Se trata, por tanto, de un concepto genuinamente dinámico, exigido de contraste intersubjetivo sociohistóricamente determinado y, en definitiva, múltiple. La educación se nos aparece como un concepto de significación múltiple, tan sólo abarcable teóricamente en el marco de un proceso dinámico de discusión racional, crítica e intersubjetiva.

Ahora bien, el concepto de educación, aún siendo esencialmente contestable, no puede significar cualquier cosa. Hemos dicho que posee una cierta contextualización social e histórica, y precisamente la historia -y principalmente la narrativa, quizá sobre todo la literatura- así como los contextos sociales dotan de pleno significado, aunque tal vez con desigual valor y rivales unos frente a otros, a nuestro concepto. No obstante, es posible obtener un cierto conocimiento o saber de la educación. Pero, ¿cómo acceder a este conocimiento dado el carácter contestable de la educación?; ¿de qué método hemos de servirnos para proceder en esta empresa?

Estas son interrogantes claves para elaborar una cierta epistemología de la teoría pedagógica, si se me permite la expresión. Porque el conocimiento del fenómeno educativo, su elaboración como saber utilizable en la práctica educativa, exige, de hecho, la aplicación de un método de trabajo particular. Y éste no es otro - no es otro en el contexto de este artículo, ya que es en el contexto de éste estudio donde se propone- que el método de recurso a las tradiciones rivales.

La cuestión que se propone es sencilla, y probablemente no muy original, aunque, así lo entiendo yo, suficientemente importante como para recordar de nuevo de qué se trata. En efecto, la educación es un concepto de una larga historia y tradición. Una tradición, como ha explicado Zubiri, es un legado, un testigo, una entrega de formas de estar vivientemente en la realidad. La tradición tiene que ver con la dimensión histórica de la vida humana así como con su condición narrativa.

Como dice Zubiri, «la tradición es continuidad de formas de vida en la realidad, y no sólo continuidad de generación del viviente (...) Gracias a estar viviendo en la realidad, el hombre llevará una vida no enclasada sino abierta a cualquier realidad. Para ello no basta con que cada hombre reciba una inteligencia sino que necesita que se den en su intelección mismas formas de vida en la realidad. El hombre no puede comenzar de cero. $Y$ en esto es en lo que consiste la tradición, y el carácter histórico de la realidad humana: en la entrega de formas de vida en la realidad, fundadas en un hacerse cargo de la realidad» (Zubiri, 1986, 201).

Se trata de formas de pensamiento, creencias, sentimientos, valoraciones, tradiciones de investigación y de prácticas que, generación tras generación, han ido elaborando y entregando las generaciones adultas a las nuevas generaciones como ayuda para que és- 
tas puedan instalarse convenientemente en la vida que van a vivir. Según ha señalado Robert Bellah, «una tradición es un modelo de interpretaciones y juicios que una comunidad ha elaborado a través del tiempo. Es una dimensión inherente a toda acción humana. Nunca se puede abandonar del todo la tradición, aunque sí se puede criticar una tradición desde el punto de vista de otra. Tradición no se opone a razón. A menudo es un debate continuo y razonado sobre el bien de la comunidad o institución cuya identidad define» (Bellah, 1989, 396).

Desde este punto de vista, una tradición incorpora una cierta teoría del conocimiento, pues esencial a ella es que el pasado nunca sea algo rechazable, de lo que podamos liberarnos, sino más bien se configura como comentario y explicación del presente. Precisamente MacIntyre subraya esta dimensión histórica, y también social, de la tradición (MacIntyre, 1987, 185 y ss.). Como teoría o forma de conocimiento, la tradición muestra que cada teoría particular, o conjunto de creencias científicas o morales, se entienden y justifican, hasta donde sea ello posible, como miembro de una serie histórica; una serie dentro de la cual lo posterior no es necesariamente superior a lo anterior, ya que una tradición puede progresar o degenerar. Lo importante es que esté en buen orden, es decir, que exista progreso. En realidad, según MacIntyre el progreso de una tradición se da desde dentro de ella misma, pero en contraste con otras tradiciones rivales. La rivalidad puede ser muy fuerte, tanta que siempre exista entre ellas algún núcleo principal intraducible. A pesar de todo, en primer lugar siempre cabe recurrir a alguna tradición extraña que sea capaz de aportar los recursos necesarios para comprender mejor las limitaciones e incoherencias de nuestra propia tradición de pensamiento, investigación y práctica. $\mathrm{Y}$ por otro lado, tal y como ha pretendido mostrar en Tres versiones rivales de la ética, la admisión de una significativa inconmensurabilidad e intraducibilidad en las relaciones de dos sistemas de pensamiento opuestos puede ser, de hecho, un prólogo para el debate racional y para un cierto tipo de debate a partir del cual una de las partes contendientes aparezca racionalmente superior a la otra (MacIntyre, 1992, 28).

Muchas de las críticas que ha recibido el recurso a tradiciones de pensamiento existentes para explicar lo actual, se han originado por la falsa creencia de que tradición se opone a razón. Esta objeción parte de un presupuesto falso, porque toda tradición implica reconocimiento, el cual es un acto eminentemente racional y cognitivo, aunque no sólo. Cada tradición tiene su propia racionalidad, una racionalidad que le es intrínseca, y de hecho sólo es posible traducir el lenguaje de una tradición rival desde el conocimiento y la racionalidad de una, dentro de la cual nos instalamos libremente.

La entrega de una tradición, o de un conjunto de ellas, implica, en cierta medida, educación. Implica una educación como iniciación, es decir: la iniciación en las formas de conocimiento público compartido. Tal iniciación es, en el fondo, un proceso de civilización y humanización (Peters, 1966; Oakeshott, 1982; Elliot, 1992).

De civilización, porque lo entregado se tiende a sintetizar, o cristalizar, en formas de conocimiento que actúan como lentes conceptuales para acceder e interpretar la realidad. Y en este sentido la educación no es otra cosa que un proceso de acceso a la realidad, introducción a la realidad. En ellas se sintetiza la historia de las contribuciones que ha realizado la humanidad, en todos los campos del saber. Y también es un proceso de humanización, porque el proceso en cuestión tiene asignado no meramente el propósito de actualizar un específico caudal de conocimientos en la joven generación, sino el objetivo de que este corpus doctrinal se incorpore vitalmente en el sujeto a fin de que sus estruc- 
turas de decisión interna -estructuras que en todo caso son estructuras morales- se conformen y permitan la realización de un proyecto personal de vida en sociedad, de carácter humanizador.

Este proceso de iniciación se da, como hemos dicho, en todas la fases del proceso educativo del sujeto, y lo mismo da que hablemos de unas u otras disciplinas o campos del saber. Pero es básico para el éxito del proceso que el sujeto sepa escuchar. Es decir, que el individuo aprenda a sujetar temporalmente su criterio de valoración al de quienes, antes que él, elaboraron la parte del saber que le es entregado. Como se ve, se trata de una sujeción temporal, por tanto nunca permanente, del criterio personal de valoración. Por consiguiente, no se trata de anular su capacidad de enjuiciamiento crítico, sino de que esta capacidad crítica vaya madurando en la medida que el saber trasmitido se vaya aprendiendo significativamente. Es este un punto crucial: el aprendizaje significativo de algo.

Se aprende significativamente cuando un sujeto es ayudado por otro para que pueda realizar una actividad mental propia en la que fundamentalmente se sacan a la luz los conocimientos y experiencias de que dispone ya como base o fundamento para acceder al sentido de lo nuevo. No hay aprendizaje significativo de algo nuevo si no tiene sentido para nosotros; y algo tiene sentido en la medida en que se vincula a lo que ya conocemos o hemos experimentado. En el aprendizaje significativo, por así decirlo, el conocimiento es construido por el sujeto, el cual no se sitúa frente al objeto de conocimiento estableciendo una mera relación individual con él. La ayuda de otro -del educador, del adulto, o de una tradición ya elaborada- es esencial para que se dispare esta actividad autoestructurante o reconstructiva.

El engarce que la tradición tiene con el conocimiento de la educación es importante. Porque la educación confiere una cierta identidad. Lo que tiene identidad es real, y justamente una tradición, como hemos dicho, es una entrega de formas o modos de estar vivientemente en la realidad. No invita al enclasamiento, sino a la apertura. Las diversas tradiciones de pensamiento pedagógico contienen precisamente el significado que el fenómeno educativo tuvo en un contexto social y cultural dado, e invitan por ello a revisar el presente a su luz. Nuestros conceptos y significados se fundamentan de este modo, de acuerdo con una cadena histórica, en conceptos y significados pasados. Construimos nuestros conceptos con la ayuda de realidades y significaciones pasadas, que guardamos en nuestra memoria histórica acumulada. Y ello responde, en suma, a la fuerte dimensión histórica de la que se alimenta y a la vez es constitutiva nuestra existencia. Por ello tienen tanto poder explicativo, comprensivo e interpretativo las narraciones, y en especial las narraciones literarias. En este punto, Rorty tiene razón en buena parte de las tesis planteadas en Contingencia, ironía y solidaridad.

Ahora bien, ¿en qué tipo de conocimiento consiste la tradición? Sin duda se trata de una forma de conocimiento práctico, como hemos apuntado. Particularmente, las tradiciones de pensamiento pedagógico son formas de pensamiento práctico, de un saber de la práctica, pues la educación es una actividad, una práctica. Tal vez, incluso, la educación no sea una práctica natural. Quiero decir que la educación no es algo que crezca naturalmente. La metáfora del crecimiento -tan utilizada en pedagogía para explicar el concepto de educación- no es útil para explicar el sentido del fenómeno formativo. En este punto, el recurso a las metáforas, a las analogías, es muy útil, pues en parte confirma 
tanto la rivalidad existente entre las tradiciones de pensamiento pedagógico como la natural contestabilidad de la educación misma.

Pues bien es relativamente fácil mostrar las implicaciones que tiene para nuestro tema el hecho de pensar la educación desde tradiciones intelectuales y pedagógicas de investigación y pensamiento. Quisiera destacar dos.

La primera es que el recurso a tradiciones rivales de pensamiento fortalece el sentido de pertenencia a una comunidad de investigación. Con ello, el mero y sencillo hecho de pertenecer a una profesión se convierte en algo más importante que la pura reivindicación de derechos laborales. Cuando se piensa la práctica profesional desde el convencimiento de la pertenencia a una tradición de pensamiento se fortalece un sentido fuerte de comunidad intelectual y de investigación.

La segunda implicación tiene que ver con la fundamentación teórica de la acción y las decisiones que se toman en la propia práctica o actividad. Desde el anterior convencimiento, la teoría pedagógica fundamenta la acción aportando una visión teórica amplia, y ampliada, de los fenómenos con los que se trabaja. Con ello se fortalece una actitud esencial para promover el sentido y el pensamiento reflexivo: la apertura de mente, la actitud que no busca tanto fundamentar las decisiones en una teoría como en una perspectiva teórica, incluso contemplativa. Es la actitud, en definitiva, que no prejuzga las fuentes teóricas de evidencia según el origen de donde provengan, sino que es receptiva, atenta a lo real, a la realidad, tanto presente, como pasada.

\section{CONOCIMIENTO PEDAGÓGICO, ACCIÓN EDUCATIVA Y EL PROYECTO MODERNO DE UNA FILOSOFÍA PRÁCTICA}

La pretensión por volver más técnicamente eficaces los procesos de intervención pedagógicos se ha ido afianzando, en las últimas décadas, al hilo de la consolidación de la educación como disciplina científica. Debido tanto al generalizado convencimiento de que la actividad educativa es en última instancia una clase de acción práctica, como a la necesidad de acompañar el notable aumento del interés por la educación con una adecuada capacitación teórico-técnica de los principales agentes que intervienen, como profesionales de la acción educativa, en su ejercicio habitual y cotidiano, lo cierto es que la tecnología educativa parece haberse convertido en una clara necesidad que no cabe razonablemente cuestionar.

Hace varias décadas la tecnología educativa, centrada en los «resultados» más que en los «procesos», era con razón criticada a causa del tipo de relación extrínseca que acababa por definir con respecto a la actividad educativa donde se aplicaba. En cambio, pocos partidarios de la tecnología de la educación negarían hoy que a ésta debe concebírsela como un proceso, más que como producto, esto es, como una «tecnología intrínseca de la educación» de índole eminentemente cognitiva e intelectual. Existen importantes razones -teóricas, filosóficas, pedagógicas, históricas, culturales, y de otra índole- capaces de explicar este cambio en el modo de concebir a la tecnología educativa y de justificarla como buena para la pedagogía pues la acción educativa no puede pretender hacer valer su importancia moral -debido a la relevancia y valor de su propósito fundamental: la progresiva ayuda a la construcción del hombre, en su calidad de humano- si al mismo tiempo se convierte sólo en un desencadenamiento de aconteci- 
mientos que se escapan a todo control racional y eficaz del agente. Aceptar, de este modo, el carácter ético de la actividad educativa exige, como primera medida, hacer visible la voluntad de eficacia y de conocimiento racional de la tarea educativa; su desencadenamiento intencional, secuencial y racional.

De acuerdo con este planteamiento inicial, constituye una urgente necesidad, derivada incluso del sentido común, deslindar claramente las críticas que pueden realizarse a los planteamientos tecnicistas de la educación del enjuiciamiento a los enfoques sustancialmente tecnológicos. Ambas perspectivas no se confunden. A pesar de todo, el empuje de determinados planteamientos tecnológicos en educación han generalizado progresivamente, como tesis incuestionablemente ciertas, diversas ideas que, tras una investigación más detenida, se revelan, al menos, suficientemente problemáticas como para convertirlas, sin más, en un lugar común.

La primera es la creencia de que el proceso de intervención educativa es una actuación práctica que, en cuanto tal, hace de sus específicos problemas cuestiones exclusivamente técnicas. Se piensa que la racionalidad pedagógica es principalmente una clase de racionalidad tecnológica que, siguiendo un esquema modificado de relación medios-fines, afirma que aquéllos mantienen una relación de dependencia lógica con las finalidades. Se generaliza la convicción de que la racionalidad pedagógica y la racionalidad educativa son formas de «racionalidad práctica», vale decir: tecnológica. No sirve, pues, distinción alguna entre formas operativas de racionalidad - una técnica y otra moral o práxica- pues con la implantación de la moderna civilización tecnológica -eminentemente cognitiva y centrada en procesos-se ha vuelto tan superflua como inútil la vieja distinción aristotélica entre pensamiento productivo y pensamiento práctico o práxico.

Cuando estas ideas se incorporan a la práctica, los hábitos profesionales y al acervo profesional de los educadores, el conocimiento pedagógico en su conjunto, y la producción de nuevo saber sobre educación (cada vez más obligado, como no podía ser menos, a atender los problemas que se presentan en la realidad), se constituye progresivamente como conocimiento estrictamente tecnológico. Con ello, la racionalidad pedagógica margina de la idea misma de «racionalidad» el tipo de saberes, bien teórico bien morales, cuyo principal objetivo es, sin embargo, volver más reflexiva y crítica la práctica educativa. Normalmente tiende a concederse, académicamente, que este tipo de saberes cumplen un papel de estricta, ambigua y superflua «formación pedagógica cultural» de los educadores, maestros o pedagogos, según se trate. Existe «concesión» de papel de formación cultural para cierto tipo de saberes. Esta «concesión» tiene diversas fuentes de origen, y de hecho, contrariamente a la determinación teórica de la tecnología educativa como proceso, en la práctica ésta no pasa de ser muchas veces más que un producto al servicio de la pretensión de medir la calidad educativa por resultados estadísticamente cuantificables. Tiende a pensarse que, en el fondo, todo enfoque crítico y reflexivo de la actividad educativa afirma la reflexividad y la crítica al precio de la eficacia pedagógica, tal y como este concepto queda definido desde posiciones tecnológicas determinadas.

Por otro lado, el ámbito de investigación centrado en la consideración de los docentes como profesionales reflexivos, desde luego ha aportado luz a la explicación de los procesos de pensamiento y toma de decisiones de los educadores, aunque en último término su mayor debilidad se encuentre, tanto en su escaso poder normativo -las investigaciones al uso son extraordinariamente descriptivas- como en ocultar lo que constituye 
la máxima preocupación de los investigadores: acabar con la división del trabajo social entre maestros y pedagogos, a quienes acusan de nuevos sofistas.

Ahora bien, a pesar de que una buena porción de este ámbito de investigación forma parte de lo que se ha dado en llamar el «contexto psicológico de la enseñanza», resulta indiscutible que sus últimos desarrollos se han venido enriqueciendo con significativas contribuciones filosóficas, mostrándose con ello el grado en que toda pretensión profesionalizadora de las distintas funciones pedagógicas - las de docencia y otras- necesariamente pasa por el redescubrimiento crítico y acentuación del carácter reflexivo de la práctica pedagógica. En este sentido, muchas de las cuestiones actualmente debatidas en relación a estos temas -desde el estudio de los procesos mentales de los educadores hasta los problemas de la relación entre la teoría y la práctica, y otros- forman parte sin ninguna duda de lo que tal vez cabría denominar como contexto de discusión filosófico de la actividad educativa.

Paralelamente a este interés profesionalizador, asimismo se ha subrayado la necesidad de capacitar tecnológicamente a las distintas figuras profesionales de la educación, con el objeto de que puedan responder en sus respectivas actuaciones con mayor eficacia y realismo a las demandas y expectativas que la sociedad en su conjunto parece tener sobre la educación. Este esfuerzo de capacitación tecnológica es concomitante de la exigencia de profesionalización en educación, como por lo demás lo sería en cualesquiera otras ocupaciones profesionales. No obstante, parece indudable que todavía existe mucho camino por recorrer hasta que dentro de la comunidad de profesionales de la educación exista verdadera eficacia y entendimiento mutuo, así como para lograr que sus procesos de razonamiento y pensamiento sean el auténtico reflejo de un cuerpo de conocimiento pedagógico científicamente constituido y válido para explicar, diagnosticar, justificar y, en último término, normativizar, la práctica educativa. Nada de ello se logrará, sin embargo, si se carece de unidad entre los profesionales de la educación, rompiéndose el monólogo colectivo que todos deploran y el distanciamiento entre la teoría y la práctica.

Sin embargo, la educación, concebida como objeto de conocimiento, ha sido pensada por algunos autores como un difícil campo de investigación en el que las expectativas habituales de la ciencia - predecir y controlar regularidades entre acontecimientos- se ven ampliamente limitadas por el hecho de que más que un puro «suceso» la educación es una acción deliberada e intencional que entraña un importante carácter ético. El estudio de la acción humana -sea ésta la educativa u otra- exige no sólo producción de generalizaciones y explicación de acontecimientos, sino, muy fundamentalmente, de justificación. El acto de justificación, o de justificar, siempre entraña un importante núcleo moral; porque justificar es hacer justicia, dar respuestas -responder-y ser responsable. Con el avance y el progreso tecnológico, esta necesidad se ha vuelto un imperativo, especialmente para el caso de una tarea práctica como la actividad educativa. En esta investigación justamente se pretende abordar el análisis de las diferentes implicaciones que la consideración de la educación como acción tiene, intentando contribuir con ello a un debate -que se desarrolla ya en las postrimerías del siglo XXen relación a la discusión de la constitución práctica de la intervención educativa y de sus principales dimensiones críticas.

En efecto, muchas disciplinas pedagógicas han partido de este dato -el carácter de «problema práctico» de la educación- a la hora de reflexionar epistemológicamente so- 
bre sí mismas y justificar sus concretas aportaciones. Ahora bien, lo cierto es que la noción de «práctica» es habitualmente entendida de diverso modo. Mientras unos entienden por «práctica» una cuestión técnica, otros pensarán que por ella debe entenderse una praxis o actividad moral de predominio de bienes y fines internos.

Como consecuencia de la pretensión de predominio de una de las dimensiones explicativas de la educación como actuación humana, y del olvido de la intrínseca complejidad que entraña la explicación de la naturaleza de la educación, se hace verdaderamente difícil encontrar un planteamiento suficientemente holístico sobre la actividad educativa que sea capaz por propia naturaleza de explicar coherentemente las diferentes dimensiones constitutivas de la educación como acción, haciendo que, en último término, consiga ser ésta una integral de actividad productiva y de acción. Constituye éste un importante núcleo de complejidad del estudio de la naturaleza de la educación.

La consideración de esta tarea como práctica reflexiva se convierte para algunos, así, en alternativa a los planteamientos que acentúan su naturaleza de actividad técnica cuando, como hemos dicho, la tecnología educativa cada vez parece mostrarse como proceso de intervención cognitivo y racional en el acto pedagógico- y ambos, también en ocasiones, muy limitadamente incluyen determinaciones éticas en la práctica de la educación, a juicio de otros autores. Por otra parte, las investigaciones referidas al pensamiento de los profesores - que pretenden restaurar en parte la tradición establecida por Aristóteles sobre la racionalidad del pensamiento práctico- tan sólo en contadas ocasiones logran recoger toda la riqueza contenida en la fundamental noción de «conocimiento práctico».

Pues bien, en este estudio se parte del reconocimiento del lugar central que este concepto ocupa en la aludida tradición, y específicamente para cualquier tentativa orientada a pensar la educación como actividad práctica. Muy concretamente se señala la urgencia de extender, para la investigación teórica y filosófica de la educación, esa nueva sensibilidad que en las últimas décadas se deja sentir en relación a los valores propios de la tradición de la filosofía práctica, y cuyas propuestas nucleares resultan pertinentes para el ámbito concreto de las ciencias de la actividad humana-como la ciencia educativa-, en las cuales muy especialmente cabe requerir que sus profesionales logren un adecuado planteamiento de las relaciones entre el pensamiento y la acción; entre la teoría y la práctica. De hecho, muchas de las tentativas actuales orientadas a la investigación de las relaciones entre la teoría y la práctica en la educación han fracasado justamente por no partir de la realidad o fenómeno que se supone pretendían explicar, comprender y dirigir: la actividad educativa.

Ahora bien, para comprender con exactitud todo lo que ello significa, hay que revisar, por una parte, la naturaleza de la cognición práctica en la educación, y, en segundo término, recordar que en el análisis de la naturaleza de la actividad educativa no se ha llegado a un acuerdo pleno acerca de lo que conlleva subrayar su carácter de «práctica».

\section{LA COGNICIÓN PRÁCTICA EN LA ACCIÓN PEDAGÓGICA: UNA REVISIÓN CRÍTICA}

Acerca del primer asunto señalaré que las actuales investigaciones, vinculadas fundamentalmente a la tradición angloamericana, que se han dedicado al estudio del pensamiento práctico de los docentes y educadores y a la investigación de sus procesos de 
deliberación, obtención de juicio y toma de decisiones, con haber contribuido notablemente a mejorar la calidad de la práctica profesional, sin embargo, han solido marginar el estudio de las bases éticas de los procesos de reflexión y cognición práctica en educación (Fenstermacher, 1988; Liston y Zeichner, 1987). También sobre este punto la investigación que presentamos intenta realizar una específica contribución, ya que pretende vincular el debate actual sobre el pensamiento práctico en educación con los fundamentos éticos -no exclusivamente deontológicos- de la dimensión reflexiva de la práctica educativa. En el marco de la actuación educativa, los razonamientos que elaboramos con la intención de guiar la acción producen un tipo de «saber normativo» que, por el mismo fin al que tienden (la dirección de la acción con fines a la vista), evocan la existencia de una actividad mental o proceso cognitivo de carácter eminentemente práctico (Manen, 1990). Evidentemente, la cognición produce un cierto tipo de saber; pero la producción de saber pedagógico (el saber referido al conocimiento «técnico», no simplemente común, de la educación) no se realiza desde un solo procedimiento: los procedimientos de obtención del saber en pedagogía, o en el contexto de la acción educativa, son múltiples.

Así pues, los procesos de razonamiento práctico -aquéllos que tienen lugar en el contexto de cualquier tarea práctica- producen, en primer lugar, un cierto saber (constituido por un cuerpo de datos e información determinada) y, en segundo término, tienen un carácter normativo en el sentido de que su elaboración se lleva a cabo con la finalidad de que el pensamiento dirija las acciones, principalmente, a través de la adopción de unas decisiones cuyo fundamento se encuentra en el saber producido por el mismo razonamiento pedagógico. Tomar una decisión es, así, tomar partido por un curso de acción determinado; esto es, hacer que la actividad se desarrolle en la línea que precisamente define la decisión adoptada. Pero una correcta elaboración del razonamiento pedagógico no se puede realizar en el vacío. Se razona a partir de un conocimiento ya adquirido, de un cuerpo de datos e información, etc. Puede decirse que el razonamiento pedagógico se organiza en torno a dos fuentes cognitivas.

En primer lugar, sobre la base del saber teórico elaborado por distintas disciplinas educativas y, en segundo término, sobre la base de los datos y el material que aporta el conocimiento de la realidad donde se actúa. El saber que producen las disciplinas educativas no es sólo teórico, en el sentido de ser el producto de especulaciones generales, sino en el sentido que la ciencia moderna atribuye a la noción misma de «teoría»: saber susceptible de establecer leyes o normas generalizables. El conocimiento de la realidad, por su parte, es más bien práctico y ajustado a determinados contextos o realidades, llamémoslas así, sociohistóricas; a veces muy subjetivo, con frecuencia condicionado por las creencias o supuestos creenciales en los que se basa el mismo acto cognitivo del educador, etc. Es un «conocimiento múltiple» y, hasta cierto punto, limitado: primero, porque sus fronteras son las que marcan la realidad a partir de la cual se obtiene conocimiento y, segundo, porque no es especulativo ni abstracto, sino que se limita a registrar lo que hay, y en todo caso a ordenar la información que se pueda extraer.

A partir de este marco de consideraciones -que replantean la cuestión de la relación entre pensamiento y acción en educación- sabemos que cuando se intentan formular modos de relacionar aquellas instancias, muchas veces las soluciones han solido venir en la dirección de reconsiderar la formación inicial de los profesionales reorientando el carácter de los conocimientos teóricos, volviéndolos más prácticos u orientados a la práctica, o acompañándolos de más ocasiones para practicarlos. En mi opinión, esta vía lleva en 
sí misma el germen de su propio fracaso, al menos en la medida que no se vea acompañada por un esfuerzo paralelo específicamente orientado a enseñar a pensar pedagógicamente al profesional en contextos de incertidumbre, lo que, a mi juicio, es tanto como iniciarles en un complejo proceso de aprendizaje de los modos de operar de la cognición práctica.

Por lo que se refiere a la segunda cuestión planteada anteriormente, me gustaría dejar claro que mi intención en esta investigación, por lo que se refiere a la determinación ética de la naturaleza práctica de la educación, no es subrayar dicho carácter a costa de la dimensión tecnológica que este quehacer posee. Más bien la cuestión será llegar a comprender el exacto lugar que las destrezas técnicas deben ocupar en la tarea. Por lo demás, pienso que una adecuada integración de la formación tecnológica en el ámbito genérico de la formación humana exige una modificación de la concepción de la propia ciencia pedagógica de modo que pueda liberarse de algunas servidumbres actitudinales y acometer la investigación de los problemas que plantea la entrada del desarrollo tecnológico en el mundo educacional. Sea como sea, una investigación sobre la naturaleza práctica de la actividad educativa mostrará la necesidad de seguir trabajando en tres ideas centrales:

1. En primer lugar, que la actividad educativa está regida por un principio de incertidumbre, es decir, por tipos de incertidumbres estructuradas y no estructuradas, siendo éstas últimas las que suponen el mayor desgaste reflexivo para el educador (Eraly, 1988; Holt, 1987; Bárcena, 1992).

2. En segundo término, que la intervención pedagógica reclama la creación de marcos interpretativos de acción, de modo que con ello se dé paso a un enfoque deliberativo y reflexivo. Esta idea puede encontrar en la noción de conocimiento práctico una de sus posibilidades de desarrollo y fundamentación más sólidas.

3. Finalmente, que como forma de actuación humana, dentro del proceso de intervención educativa, los acontecimientos y situaciones que se crean se encuentran en estrecha dependencia del tipo de decisiones pedagógicas que los educadores toman. Como forma de actuación práctica, la tarea educativa es una empresa moral, y cabe por tanto explicar y justificar la posibilidad de su consideración profunda e inherentemente ética (Bárcena, 1989, 1991a; Bárcena, Gil y Jover, 1992 y 1993).

En las últimas décadas asistimos a un empeño teórico básicamente centrado en el establecimiento de la racionalidad y la acción verdaderamente humanas. Ambos conceptos son centrales para la investigación del carácter práctico de la actividad educativa, pues, en cuanto tal, su racionalidad específica es la racionalidad práctica, y en cuanto actuación práctica, una acción en sentido estricto: intencional y deliberada.

Un reflejo en nuestro ámbito de este movimiento de rehabilitación de la razón práctico lo encontramos en las investigaciones realizadas por Gauthier (1963) sobre la naturaleza de los «problemas prácticos», que han influido en la elaboración de un enfoque práctico-moral para el estudio del currículo, principalmente a través del desaparecido Schwab (1985), y los posteriores desarrollos de William A. Reid (1978). Para éste último, los problemas curriculares se constituyen básicamente como asuntos prácticos inciertos y singulares estrechamente relacionados con la deliberación y la reflexión, y sus notas características principales son las siguientes:

a) En primer lugar, son cuestiones que han de ser respondidas de acuerdo a las exigencias de una situación concreta y particular, aún cuando la respuesta reclamada sea no hacer nada. 
b) En segundo término, este tipo de problemas exige un continuo proceso de toma de decisiones, ya que a toda acción precede una decisión y a ésta la deliberación. Pero es distintivo de ellos que tales decisiones se apoyen en inciertos fundamentos. No existe una firme base que nos asegure una decisión correcta. Justamente por ello, responder a las demandas que los problemas prácticos reclaman precisa tener en cuenta un importante número de elementos y factores colaterales.

c) En tercer lugar, todo problema práctico se presenta como caso singular y único que demanda una respuesta ad hoc. En esta clase de cuestiones con frecuencia nuestras decisiones se enfrentan ante la necesidad de elegir entre fines y valores en conflicto, y la dificultad, o casi imposibilidad, de poder predecir un resultado seguro y exitoso.

En una palabra, un problema práctico está inmerso en la esfera de lo incierto y de la inseguridad. Y es justamente esta razón la que explica que las zonas indeterminadas de la práctica educativa supongan para el educador un gran desgaste reflexivo, deliberativo y de enjuiciamiento responsable (Schön, 1983; 1987; Jackson, 1986; Powell, 1980; Polanyi, 1989; Scheuerl, 1984; Tvershy y Kahnemann, 1974; Vickers, 1983; Zabalza, 1987; Zaret, 1981). Stenhouse (1984) había reconocido este último aspecto también en sus trabajos. Pese a las innumerables interrogantes y dudas que ha planteado la defensa que este autor realizó en su Humanities Curriculum Project del conocido «principio de neutralidad de procedimiento», inspirado en los principios de procedimiento formulados previamente por Peters (1966), lo cierto es que el «modelo de proceso» por él ideado -como alternativa al «modelo de objetivos de conducta», de carácter más bien tecnicista- es esencialmente crítico y profesionalizador para los profesores.

Desde el ángulo filosófico, la propuesta hermenéutica de Gadamer (1981 a; 1981 b; 1977 ; 1966) y la teoría crítico-comunicativa de Habermas $(1989 ; 1987)$, también se han dejado sentir en la literatura pedagógica especializada, tanto en los enfoques deliberativo-reflexivos de la enseñanza y de la formación del profesorado (Angulo Rasco, 1989; Dewey, 1989; Eisner, 1979 y 1983; Floren y Clark, 1988; Gimeno, 1988; Harris, 1986; Kniter, 1985; Reid, 1978, 1979 y 1981; Roby, 1985; Rubin, 985; Trumbull, 1983; Westbury y Wilkof, 1978; Zeichner, 1987), como en la formulación de una teoría crítico-interpretativa de la práctica educativa (AAVV, 1986; AAVV, 1989; Beyer, 1988; Bredo y Feimber, 1982; Carr, 1990; Carr y Kemmis, 1988; Grundy, 1991; Liston y Zeichner, 1987; Popkewitz, 1988). A modo de resumen, puede advertirse la existencia de una triple vía de investigación -que entre sí presentan profundas concomitancias- dentro del marco del movimiento de rehabilitación de la razón práctica.

En primer lugar son destacables los estudios relacionados con el análisis del pensamiento de los profesores, toma de decisiones y elaboración de juicios en marcos pedagógicos de actuación prefigurados, aunque dotados de zonas de incertidumbre e indeterminación. Los principios rectores de este campo son, sustancialmente, tres: a) la convicción de que lo que los profesores y otros educadores hacen está fuertemente influenciado por su pensamiento, que a menudo es tácito y poco estructurado; b) la convicción de que la práctica de la enseñanza, y en general toda actividad educativa, se basa en un cuerpo más o menos sistemático de conocimientos, actitudes, destrezas y creencias; y c) la convicción de que, como profesional, el educador es un agente reflexivo llamado a tomas de decisiones y elaborar juicios de naturaleza moral (Calderhead, 1987; Cruickshank, 1981; Elbaz, 1983; Grant, 1984; Halhes y Olson, 1984; Smith, 1987; Villar Angulo, 1988; Wittrock, 1989). 
En segundo término, la teoría y la filosofía de la acción humana ha venido proporcionando interesantes desarrollos en orden a una comprensión más real de la práctica educativa. Destacan aquí la noción de «problema práctico»-en la línea del mencionado Gauthier (1963) - y también las investigaciones analíticas encaminadas a la determinación de la «red conceptual de la acción». Este tipo de trabajos podrían ser extraordinariamente aprovechables para la exploración y caracterización puramente descriptiva de «la educación como acción», en el sentido de describir y analizar los discursos en los cuales el hombre -en nuestro caso el educador- «dice su hacer», y haciendo abstracción de la calificación moral de la misma. Tal es la propuesta, previa a la misma ética, que para el ámbito general de la filosofía de la acción hace Paul Ricoeur (1988).

Dentro de una línea de trabajo político-sociológica, son asimismo innegables las aportaciones que sobre la pareja de nociones «acción-discurso» y sobre el concepto de «acción social» han realizado, respectivamente, H. Arendt (1974) -en «La condición humana»- y Max Weber (1984), en sus investigaciones metodológicas sobre esta cuestión. Interesantes comentarios aplicados a nuestro campo podemos encontrar en trabajos como el de J. Masschelein (1990).

La investigación de la naturaleza de las profesiones recientemente ha llegado a enmarcar las diferentes actividades sociales según el tipo de operaciones mentales, destrezas y específica racionalidad -técnica o práctica- más apropiada a los objetivos que persiguen. Destacan aquí los estudios de Schön $(1987$; 1983), sobre el concepto de «reflexión en la acción» y las «zonas indeterminadas de la práctica profesional», y los de Argyris (1987) sobre la noción de «action science».

Finalmente, a todo lo anterior habría que añadir algunos sugerentes desarrollos que acentúan la necesidad de pensar la educación como arte, sea en la dirección de los planteamientos de A. Tom (1984) -que piensa en la enseñanza como en un «oficio moral» (moral craft) - o en la de Luis J. Rubin (1985), que concibe al profesor como un «artista» comprometido en la elección de fines educativos dotados de un valor superior, en el uso de procedimientos imaginativos e innovadores para alcanzarlos y con el cultivo personal de ciertas habilidades y destrezas que faciliten esta tarea.

\section{LA ESTRUCTURA PRÁCTICA DE LA ACTIVIDAD EDUCATIVA}

Una de las conclusiones a que conduce el estudio de lo más significativo de esta literatura es que las actividades del tipo de tarea educativa se encuentran regidas, como antes se dijo, por el principio de incertidumbre, y que su dirección precisa siempre la mediación de un tipo particular de reflexión: la deliberación moral. De acuerdo con este punto de vista, y también desde una perspectiva profesionalizadora de las actividades relacionadas con la educación, se ha venido reclamando para una adecuada dirección de la práctica educativa una mayor acentuación de los procesos reflexivos de deliberación junto a la conformación moral de la propia acción educativa. Es decir, la convicción de que el educador, como profesional, es un agente reflexivo que no puede separarse de la creencia de que, simultáneamente, es un agente moral cuyas decisiones pedagógicas y juicios educativos deben desplegarse sobre la base de una estructura cognitiva de moralidad firmemente asentada, esto es, sobre la base de una estructura ética general. 
Pues bien, su desarrollo en los educadores supone y exige la simultánea mejora de sus procesos de reflexión, de comprensión moral de las situaciones educativas y de conformación ética -en base a principios morales de procedimiento- de las acciones pedagógicas.

De acuerdo con estas ideas, se puede decir que donde verdaderamente acaba dilucidándose la condición de actividad y de acción de la educación es en el análisis de su condición de actuación humana. Así pues, de lo que se trata es de situar convenientemente los dos pilares de la constitución práctica de la tarea educativa -el ser actividad (técnica) y acción (práxica)- en su lugar adecuado.

En efecto, la educación puede ser concebida como un objeto de estudio y como una específica clase de actuación humana. Como «objeto de estudio», la educación se caracteriza por una cierta complejidad teórica y práctica. Y en este sentido, afirmamos como principio básico que la «educación» es un difícil campo de estudio e investigación en el que las expectativas habituales de la ciencia - predecir y controlar regularidades entre acontecimientos- se ven ampliamente limitadas, pero no anuladas, porque la educación, más que un mero «suceso» es una «acción» cuya finalidad es configurar intencionalmente situaciones pedagógicas (Widlak, 1984). Tal configuración no es posible realizarla al modo en que desde la investigación científica se aplica un cierto saber o cuerpo de conocimientos para corroborar hipótesis. Como «actuación humana», la educación tiene un indudable carácter práctico, y así suele referirse uno a la educación como a una «práctica social», una «práctica política», una «práctica moral», una «práctica técnica», etc, dependiendo, en cada caso, del énfasis que se desee subrayar. En cualquier caso, en tanto que actuación, se constituye en una «práctica plural».

Toda práctica posee, al menos, tres rasgos esenciales: bienes internos, que permiten sostener al agente en la búsqueda de los fines propios de la tarea; determinadas reglas (en parte «básicas», porque explican el funcionamiento de la actividad; y en parte «estratégicas» ya que muestran cómo obtener éxito en ella, por ejemplo combinando reflexivamente determinadas reglas básicas); y, finalmente, modelos específicos de calidad, excelencia y autoridad (MacIntyre, 190; 1988; 1987; Pearson, 1989; Bárcena, 1989 у 1991b).

Concebida en estos ámbitos, la actuación educativa puede ser explicada atendiendo, al menos, a cuatro dimensiones fundamentales, que a su vez se estructuran, por pares, en dos ámbitos estructurales distintos (el ámbito productivo de actividad y ámbito práxico de acción). A tales dimensiones las llamaré «dimensiones críticas de la educación», ya que es crucial atenderlas para tener éxito y a la vez dar sentido humano a la actuación educativa.

En efecto, la actuación educativa es tanto «actividad» como «acción», las cuales se ensamblan estructuralmente en virtud del principio de responsabilidad, y en torno a las cuales se estructuran las siguientes cuatro dimensiones: la dimensión tecnológica, en la que destaca el principio de eficacia; la dimensión artística, en la que destaca el principio del tacto; la dimensión reflexiva, que se relaciona con el principio de incertidumbre; y la dimensión ética, que se fundamenta en el principio de libertad. En principio, la primera tiene que ver con el «ámbito productivo de actividad» de la educación, y las restantes con su «ámbito práxico de acción»; y digo «en principio» porque sólo abstrayéndolas es posible distinguirlas dibujando sus contornos y perfiles separadamente. En la realidad, la tecnología dice estrecha relación a procesos de cognición práctico-reflexiva, y la acción 
reflexiva a la idea del tacto pedagógico - que ya para Herbart era un mediador entre teoría y práctica. Por lo mismo, sus respectivos principios reguladores funcionarán, unos frente a otros, como coprincipios (ver figura 1).

De acuerdo con ello, puede afirmarse que el conocimiento de la educación presenta una pluralidad de formas, pues el saber sobre educación tiene que ver satisfechas las exigencias que demandan estas cuatro dimensiones. El conocimiento pedagógico, en efecto, se puede producir desde distintas clases de acciones, desde distintas formas de pensamiento y a partir de distintos actos de contemplación. De este modo, y salvando lo dicho hasta ahora, cabe hablar de un saber teórico-tecnológico (que explica, diseña y planifica); de un saber práctico reflexivo (que capacita para la deliberación, la elaboración de juicios y la toma de decisiones prácticas); y de un saber prudencial (que busca fundamentar éticamente la actuación educativa).

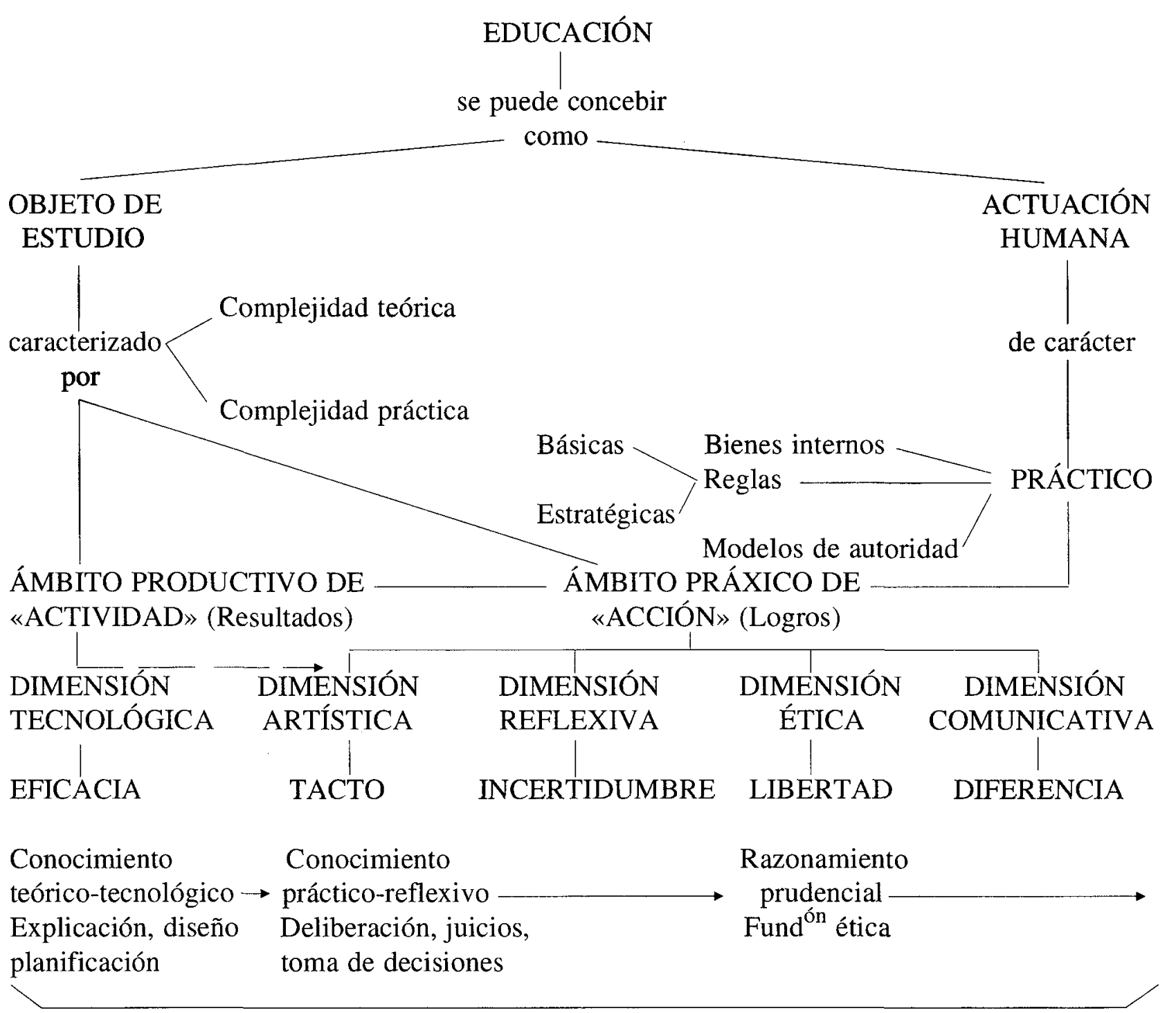

Figura 1.

Así pues, cuando se afirma que la acción educativa tiene un carácter práctico, parte de lo que se puede pretender querer decir con ello es que se trata principalmente de la 
intervención en una cierta realidad -la del educando- en la que juegan un papel esencial los procesos reflexivos de deliberación, la elaboración de juicios y la toma de decisiones. Desde este punto de vista, considerada como un problema a resolver, la acción educativa, o pedagógica, es un problema práctico; esto es, el tipo de problema que se resuelve mediante la aplicación de determinados conocimientos y técnicas de modo primordialmente reflexivo y deliberando sobre las situaciones donde se actúa, estimando diversas realidades, o juzgándolas, tomando decisiones y haciendo determinadas cosas; actuando, en definitiva.

En resumen: los profesionales de la educación -maestros, docentes, en general los educadores- intervienen dentro de los procesos que les ocupan de distinta manera y moviéndose, por así decirlo, en planos de actuación diversos. Dicho con otras palabras: en su actuación pueden mostrar un grado de competencia que no es posible calificar solamente en los términos de una «competencia técnica». Indudablemente, en ocasiones actúan de una forma realmente técnica (ajustando medios a fines dados), pero otras veces lo hacen de una forma reflexiva, y en otras de modo que, quizá precisamente por haberlo hecho de este último modo, conduciéndose a partir de criterios o principios éticos. A estas diversas formas de actuación podemos denominarlas genéricamente «dimensiones explicativas de la acción pedagógica».

\section{BIBLIOGRAFÍA}

AAVV (1986). Tecnología y educación. CEAC, Barcelona.

AAVV (1986). «Teachers, teching and teacher education». Harvard Educational Review, 56:4 (Número monográfico).

AAVV (1989). «Critical reflection in teacher education. Practices and problems». Journal of Teacher Education, 40:2 (Número monográfico).

American Association of COLleges for TEACHER EdUCATION (1988). Teacher education pipeline, AACTE, Washington, D. C.

AMERICAN ASSOCIATION OF UNIVERSITY PROFESSORS (1988). «Mastering the academic markplace. The annual report of the economic status of the profession». Academe, 74:2, pp. 3-16.

ANDREWS, K. R. (ed.) (1989). Ethics in practice. Managing the moral corporation, Harvard Business School Press, Boston.

ANGUlo RASCO, F. (1989). «Hacia una nueva racionalidad educativa. La enseñanza como práctica». Investigación en la Escuela, $\mathrm{n}^{\circ}$ 7, pp. 23-35.

ARANGUREN, J. L. (1976). «El ethos, carácter o personalidad moral», en Ética, pp. 292-297, Alianza, Madrid.

ARENDT, H. (1974). La condición humana, Seix Barral, Barcelona.

ARISTÓteles (1985). Ética a Nicómaco, Centro de Estudios Constitucionales, Madrid.

Aristóteles (1983). Política, Centro de Estudios Constitucionales, Madrid.

ARNOLD, P. J. (1990). «Educación, conocimiento práctico y movimiento». En Educación física, movimiento y currículum, pp. 29-43, Morata, Madrid.

ARGYRIS, C. y otros (1987). Action science, Jossey-Bass, San Francisco. 
ARGYRIS, C. y SCHÖN, D. A. (1974). Theory in practice. Incressing professional effectiveness, Jossey-Bass, San Francisco.

ASHCROFT, K. y GRIFFIFHTS, M. (1989). «Reflective teachers and reflective tutors». Journal of Education for Teaching, 51:1, pp. 35-52.

ASSOCIATION OF TEACHER EDUCATORS (1986). Visions of Reform: Implications for the education profession. (Reston, Va., Association of Teachers Education).

BALL, T. (ed.). Political theory and praxis, University of Minnesota Press, Mineápolis.

BÁrCENA, F. (1989). «Explicación de la educación como práctica moral». Revista Española de Pedagogía, XLVII: 183, mayo-agosto, pp. 245-278.

BÁrCENA, F. (1990). Los límites de la Filosofía de la Educación como saber práctico, en VARIOS. Filosofia de la Educación hoy, II, pp. 247-252, UNED, Madrid.

BÁrCENA, F. (1991a). La consistencia ética de la profesión educativa, Pad'e, nº 2, pp. 5-30.

BÁrCENA, F. (1991b). «Teoría de la Educación y conocimiento práctico. Sobre la racionalidad práctica de la práctica educativa». Revista Complutense de Educación, vol. 2, $\mathrm{n}^{\circ} 2$, pp. 221243.

BÁrCENA, F. (1992). «El principio de incertidumbre en la educación», Diálogo Filosófico, I: 22, pp. 59-69.

BÁRCENA, F. (1993). «El tratamiento de la incertidumbre en la enseñanza reflexiva. Bases para una teoría del juicio pedagógico», Revista de Educación, n 293, enero-abril (en prensa).

BÁrCENA, F.; GIL, F. y Jover, G. (1991). «La dimensión ética de la actividad educativa. Notas críticas para el replanteamiento de un problema», Bordón, 43:3, pp. 259-269.

BÁrCENA, F; GIL, F. y Jover, G. (1993). «The ethical dimension of teaching: Review and Proposal», Journal of Moral Education, 22:1. Número monográfico sobre «Ethical dimensions in teaching and teacher's professional morality» (en prensa).

BARON, F. (1988). Thinking and deciding, Cambridge University Press, Nueva York.

BEINER, R. (1983). Political judgment, Methuen, Londres.

BELLAH, R. y otros (1989). Hábitos del corazón, Alianza, Madrid.

BeLth, M. (1971). La educación como disciplina científica, El Ateneo, Buenos Aires.

Bernstein, R. J. (1979). «Acción, conducta e investigación. Peirce y Dewey», en Praxis y acción, pp. 173-235, Alianza, Madrid.

- (1983). Beyond objetivism and relativism, Science, hermeneutics and praxis, University of Pensylvania Press, Filadelfia.

- (1985). The restructuring of social and political theory, Methuen, Londres.

BEYER, L. E. (1988). Knowing and acting, The Falmer Press, Londres.

- (1988). «La reconstrucción del conocimiento y de los estudios educativos», Revista de Educación, $\mathrm{n}^{\mathrm{o}} 286$, pp. 129-150.

Bонм, W. (1982). «¿Es posible profesionalizar la actividad del maestro?», en La educación del hombre, pp. 87-98, Docencia, Buenos Aires.

BREDo, E. y FEINBERG, W. (1982). Knowledge and values in social and educational research, Temple University Press, Filadelfia.

BrezinKA, W. (1990). Conceptos básicos de la ciencia de la educación, Herder, Barcelona. 
BUBner, R. (1985). Azione, linguaggio e ragione, Il Mulino, Bolonia.

CALDERHEAD, J. (1987). Exploring teacher's thinking, Cassell, Londres.

CARR, D. (1981). «Knowledge in practice», American Philosophical Quarterly, 18:1, pp. 53-61.

- (1986). «Education, professionalism and theories of teaching», Journal of Philosophy of Education, 20:1, pp. 113-122.

CARR, W. (1987). «What is an educational practice?». Journal of Philosophy of Education, 21:2, pp. $163-175$.

- (ed.) (1989). Quality in teaching, Arguments for a reflective profession, The Falmer Press, Londres.

- (1989). «Prólogo» a RoZADA, J. M. y otros, Desarrollo curricular y formación del profesorado, pp. 19-30, Cyan, Oviedo.

- (1990). Hacia una ciencia crítica de la educación, Laertes, Barcelona.

CARR, W. y KEMMIS, S. (1988). Teoría crítica de la enseñanza, M. Roca, Barcelona.

CARR-SAunders, A. M. (1933). The professions, Clarendon, Oxford.

CAStillejo, J. L. (1987). Pedagogía tecnológica, CEAC, Barcelona.

- (1987). «Investigación y acción educativa: un modelo de integración de la teoría y la práctica», en J. L. y otros, CASTILleJo, Investigación educativa y práctica escolar, pp. 9-31, Santillana, Madrid.

CHARLeS, D. (1984). Aristotle's philosophy of action, Duckworth, Londres.

COE, R. M. (1984). «La profesionalización de la medicina», en Sociología de la medicina, pp. 218-251, Alianza, Madrid.

Coll, C. (1990). Aprendizaje escolar y construcción del conocimiento, Paidós Ibérica-MEC, Barcelona.

Colom, A. (1986). «Pensamiento y teoría de la educación», en AAVV (1986). Tecnología y educación, pp. 13-32, CEAC, Barcelona.

CortinA, A. (1985). Razón comunicativa y responsabilidad solidaria, Sígueme, Salamanca.

- (1986). Ética mínima, Tecnos, Madrid.

- (1989). «La ética discursiva», en CAMPS, V. (ed.). Historia de la Ética, vol. III, Crítica, Barcelona.

- (1991). Ética sin moral, Tecnos, Madrid.

Cruickshank, D. (1981). Reflective teaching, Phi Delta Kappa, Bloomington.

DAlle FratTE, G. (1988). La decisione in pedagogia, Armando, Roma.

Damiano. E. (1988). L'insegnamento come azione. Il Quadrante Scolastico, 38, pp. 23-48.

DANIELS, L. B. (1975). «What is the language of the practical?». Curriculum Theory Network, 4, pp. 237-261.

DEwey, J. (1950). Lógica. Teoría de la investigación, FCE, México.

- (1952). En busca de la certeza, FCE, México.

- (1966). Naturaleza humana y conducta, FCE, México. 
- (1980). Democracia y educación, Losada, Buenos Aires.

- (1989). Cómo pensamos, Paidós Ibérica-MEC, Barcelona.

EISNER, E. W. (1979). «On the art of teaching», en The educational imagination, pp. 153-167, Macmillan, MacMillan.

- (1983). «The art and craft of teaching», Educational Leadership, 40:4, pp. 4-13.

ElbaZ, F. (1983). Teacher thinking. A Study of practical knowledge, Cromm Helm, Londres.

Elliot, J. (1990). La investigación-acción en educación, Morata, Madrid.

ESCÁMEZ, J. (1983). «Educación y normatividad», en AAVV. Teoría de la Educación, pp. 65-77, Límites, Murcia.

ESCÁMEZ, J. (1990). «La filosofía de la Educación como praxeología educativa», en La Filosofía de la Educación hoy. Actas del Congreso Internacional de Filosofía de la Educación, vol. I, pp. 109-120, UNED, Madrid.

Escolano Benito, A. (1980). «Diversidad de profesiones y actividades educativas», Revista Española de Pedagogía, XXXVIII:147, pp. 83-98.

ERALY, A. (1989). «La structuration de l'entreprise», La rationalité en action, Université Libre de Bruxelles, Bruselas.

Esteve, J. M. (1987). El malestar docente, Laia, Barcelona.

- (1988). «El estrés de los profesores: propuestas de intervención para su control», en A. VILLA (coord.). Perspectivas y problemas de la función docente, Narcea, Madrid.

FENSTERMACHER, G. D. (1988). «Tres aspectos de la filosofía de la investigación sobre la enseñanza», en M. C. WitTrock (ed.). La investigación de la enseñanza, I, pp. 150-180, Paidós-MEC, Barcelona.

FERRER, U. (1990). Perspectivas de la acción humana, PPU, Barcelona.

Floden, R. E. y ClARK, Ch. M. (1988). Prepairing teachers or uncertainty, Teachers College Record, 89:4, pp. 505-524.

Fullat, O. (1984). Verdades y trampas de la pedagogía, CEAC, Barcelona.

GADAMER, H. G. (1967). «Theory, technology, practice, The task of the science of man». Social Research, 44, pp. 529-561.

- (1977). Verdad y Método, Sígueme, Salamanca.

- (1981). «QQué es la praxis? Las condiciones de la razón social», en La razón en la época de la ciencia, pp. 41-57, Alfa, Barcelona.

- (1981). «Hermenéutica como filosofía práctica» en La razón en la época de la ciencia, pp. 59-81, Alfa, Barcelona.

GAlliE, W. B. (1967). The idea of practice, Proceedings of the Aristoteliam Society, LXVIII, pp. 63-86.

GAUTHIER, D. P. (1978). «Practical problems», en A. HARNETT y M. NAISH (eds.). Theory and the practice of Education, vol. I, pp. 18-24, Heinemann, Londres.

- (1963). Practical reasoning, Oxford University Press, Oxford.

GIMENO SACRISTÁN, J. (1985). Teoría de la enseñanza y desarrollo del currículum, Anaya, Madrid. 
- (1988). El currículum: una reflexión sobre la práctica, Morata, Madrid.

- (1989). «Profesionalidad docente, currículum y renovación pedagógica», Investigación en la Escuela, $\mathrm{n}^{\circ}$ 7, pp. 3-21.

GOODland, J. I.; SODER, R. y SiROTNIK, K. (eds.) (1988). The moral dimensions of teaching, Jossey-Bass, San Francisco.

GoRdon, P. y otros (1985). Is teaching a profession?, Bedford Ways Papers, Londres.

GOYeTtE, G. y LeSSARD-HEBERT, M. (1988). La investigación-acción, Laertes, Barcelona.

GrimmetT, P. y ERICKSON, G. (eds.) (1988). Reflection in Teacher Education, Teachers College Press, Nueva York.

Grant, L. A. (ed.) (1984). Prepairing for reflective teacher, Allyn and Bacon, Boston.

GREEN, TH. (1976). «Teacher competence as practical rationality», Educational Theory, 26:3, pp. 249-258.

- (1985). «The formation of conscience in an age of technology», American Journal of Education, 94:1, pp. 1-33.

- (1988). «The economy of virtue and the primacy of prudence», American Journal of Education, 96:2, pp. 127-143.

Grundy SH. (1991). Producto o praxis del currículum, Morata, Madrid.

Habermas, J. (1989). «Aspectos de la racionalidad de la acción», en Teoría de la acción comunicativa. Complementos y estudios previos, pp. 369-398, Cátedra, Madrid.

- (1987). Teorías y praxis, Tecnos, Madrid.

HALKES, R. y Olson, J. K. (eds.) (1984). Teacher Thinking, ISATT, Lisse.

Hamm, C. M. (1989). «An analysis of the concept of education», en Philosophical issues in education. An introduction, pp. 29-43, The Falmer Press, Londres.

HARNETT, A. y NAISH, M. (eds.) (1978). Theory and the practice of education, Heinneman, Londres.

HARRIS, H. B. (1986). «Communicating the character deliberation», Journal of Curriculum Studies, 18:2, pp. 115-132.

HaUerwas, S. (1981). A community of character, University of Notre Dame Press, Notre Dame.

Heller, A. (1988). Más allá de la justicia, Crítica, Barcelona.

Hennis, W. (1973). Política y filosofía práctica, Paidós, Buenos Aires.

HiRsT, P. (1982). «Professional Authority; Its foundations and limits», British Journal of Educational Studies, m. 30:2, pp. 71-89.

HIRST, P. (ed.) (1983). Educational theory and its foundations disciplines, RKP, Londres.

Hogan, P. (1990). «What makes practice educational?», Journal of Philosophy of Education, $24: 1$, pp. $15-26$.

HoLT, M. (1987). Judgment, planning and educational change, Harper and Row, Londres.

HotToIS, G. (1983). «Aspects d'une philosophy de la technique», en J. SOJCHER y G. HotToIS (eds.). Morale et enseignement, pp. 53-66, Université de Bruxelles, Bruselas.

Houston, W. R. (ed.) (1990). Handbook of Research on Teacher Education, Macmillan, Nueva York. 
HoYLE, E. (1980). «Professionalization and desprofessionalization in education», en H. HoYLE (ed.). World Yesrk Book of Education 1980: Professional Development of Teachers, pp. 42-56, Kogan Page, Nueva York.

- (1985). «The professionalization of teachers. A paradox», en P. GoRdon (ed.). Is teaching a profession?, pp. 44-54, Bedford Ways Papers, Londres.

INCIARTE, F. (1974). El reto del positivismo lógico, Rialp, Madrid.

JACKSON, PH. (1991). La vida en las aulas, Marova, Madrid.

- (1986). The practice of teaching, Teachers College Press, Nueva York.

JoNAS, H. (1984). The imperative of responsability, University of Chicago Press, Chicago.

KANT, I. (1984). Teoría y praxis, Leviathan, Buenos Aires.

KEKES, J. (1989). «Good Judgment», en Moral tradition and individuality, pp. 128-140, Princeton University Press, Nueva Jersey.

Kelly, A. V. (1989). The curriculum. Theory and practice, Paul Chapman, Liverpool.

KemMis, S. (1988). El currículum: más allá de la teoría de la reproducción, Morata, Madrid.

KEMMIS, S. y MCTAGGART, R. (1988). Cómo planificar la investigación-acción, Laertes, Barcelona.

KNIGHT, P. y SMITH, L. (1989). «In serch of good practice». Journal of Curriculum Studies, 21:5, pp. 427-440.

KNITTER, W. (1985). «Curriculum deliberation. Pluralism and the practical». Journal of Curriculum Studies, 17:4, pp. 383-396.

Kratochwil, L. (1987). «El concepto de la educación desde la perspectiva de la teoría de la acción», Educación (Tubinga), vol. 35, pp. 98-120.

KrUSKaL, W. (ed.) (1982). The social sciences, University of Chicago Press, Chicago.

Kultgen, J. (1988). Ethics and professionalism, University of Pennsylvania Press, Filadelfia.

LADRIERE, J. (1977). El reto de la racionalidad, Sígueme, Salamanca.

LANGFORD, G. (1989). «The idea of a social practice», en W. CARR (ed.). Quality of teaching. Arguments for a reflective profession, pp. 21-34, The Falmer Press, Londres.

- (1978). Teaching as a profession, Manchester University Press, Manchester.

- (1973). «The concept of education», en G. LANGFORD y D. J. O'CONNOR (eds.). New essays in the philosophy of education, pp. 3-32, RKP, Londres.

LISTON, D. P. y ZEICHNER, K. M. (1991). Teacher education and the social conditions of schooling, R. K. P., Nueva York.

- (1987). Critical pedagogy, American Journal of Education, 169:3, pp. 117-137.

- (1987). «Reflective teacher education and moral deliberation», Journal of Teacher Education, 38:6, pp. 2-8.

LOUCH, A. R. (1969). Explanation and human action, University of California Press, Berkeley.

MacinTYRE, A. (1987). Tras la virtud, Crítica, Barcelona.

- (1988). Whose justice? Which rationality?, Duckworth, Londres.

- (1990). Three rival versions of moral enquiry, Notre Dame Press, Notre Dame. 
MCCARTHY, TH. (1987). «Sobre la lógica del discurso práctico», en La teoría crítica de J. HABERMAS, pp. 359-384, Tecnos, Madrid.

Macdonald, J. P. (1992). Teaching. Making Sense of an Uncertain Craft, Teachers College, Nueva York.

MANEN, M. VAN (1977). «Linking ways of knowing with ways of being practical», Curriculum Inquiry, 6:3, pp. 205-228.

- (1990). Researching lives experience, Human science for an action sensitive pedagogy, The Falmer Press, Londres.

MaRañón, G. (1981). Vocación y ética, Austral, Madrid.

Masschelein, J. (1990). «L'éducation comme action, A propos de la pluralité et de la naissance», Orientamenti Pedagogoci, XXXVII:4 (229), pp. 760-772.

MinisTeRIO de EdUCACIÓN y Ciencia (1989). Libre Blanco para la Reforma del Sistema Educativo Español, Secretaría de Publicaciones del MEC, Madrid.

Mitchan, C. (1989). ¿Qué es la filosofía de la tecnología?, Anthropos, Barcelona.

Moore, T. W. (1974). Introducción a la teoría de la educación, Alianza, Madrid.

Muguerza, J. (1986). La razón sin esperanza, Tecnos, Madrid.

NANNI, C. (1989). «I valori e l'azione in educazione», Il Quadrante Scolastico, 42, pp. 8-22.

NovaK, J. D. y Gowin, D. B. (1988). Aprendiendo a aprender, M. Roca, Barcelona.

NovoA, A. (1987). Le temps de professeurs, vol. 1, Instituto Nacional de Investigaçao, Lisboa.

OAKESHOTT, M. (1982). «La educación: el compromiso y su frustración», en R. F. DEARDEN y otros (eds.). Educación y desarrollo de la razón, pp. 35-60, Narcea, Madrid.

- (1962). Rationalism in politics, Methuen, Londres.

OLSON, J. (1987). «El cambio en educación, ¿Por qué persiste todavía la racionalidad técnica?». Revista de Innovación e Investigación Educativa, 3, pp. 103-110.

Polin, R. (1968). «Théorie et pratique», en Ethique et politique, pp. 1-38, Sirey, Paris.

Powell, A. G. (1980). The uncertain profession, Harvard University Press, Cambridge.

PEARSON, A. T. (1989). The teacher, Theory and practice in teacher education, RKP, Londres.

PETERS, R. S. (1966). Ethics and education, G. Allen and Unwin, Londres.

- (1969). «¿En qué consiste el proceso educacional?», en El concepto de educación, pp. 13-46, Paidós, Buenos Aires.

- (ed.) (1973). Filosofía de la Educación, FCE, México.

- (1980). Authority, responsibility and education, G. Allen and Unwin, Londres.

POLANYI, M. (1989). La logique de la liberté, PUF, Paris.

PoPKEwITZ, T. S. (1988). Paradigma e ideología en investigación educativa, Mondadori, Madrid.

- (1990). Formación de profesorado. Tradición, teoría y práctica, Universidad de Valencia, Valencia.

Quintanilla, M. A. (1989). Tecnología: un enfoque filosófico, Fundesco, Madrid.

Quintanilla, M. A. y VARgas-MachuCA, R. (1989). La utopía racional, Espasa, Madrid. 
REID, W. A. (1978). Thinking about the curriculum, RKP, Londres.

- (1979). Practical reasoning and Curriculum theory, Curriculum Inquiry, 9:3, pp. 186-207.

- (1981). «The deliberative approach to the study of curriculum and its relation to critical pluralism», en M. LAWN y L. BARTON (eds.). Rethinking curriculum studies, Croom Helm, Londres.

- (1983). Curriculum design and moral judgement, Cambridge Journal of Education, $\mathrm{n}^{\circ}$ 1, pp. 3-7.

RESCHER, N. (1980). La primacía de la práctica, Tecnos, Madrid.

REYNOLDS, M. C. (ed.). Knowledge base for the beggining teacher, Pergamon Press, Oxford.

RICH, J. M. (1984). Professional ethics of education, Ch. C. Thomas, Illinois.

Ricoeur, P. (1988). El discurso de la acción, Cátedra, Madrid.

RoBy, TH. W. (1985). Habits impending deliberation Journal of Curriculum Studies, 17-1, pp. $17-35$.

RUBIN, L. J. (1985). «Teaching as art», en Artistry in teaching, pp. 13-96, Random House, Nueva York.

RYLE, G. (1967). El concepto de lo mental, Paidós, Buenos Aires.

SARRAMONA, J. (1990). Tecnología Educativa (Una valoración crítica), CEAC, Barcelona.

SCHEFFlER, I. (1970). «La enseñanza», en El lenguaje de la educación, pp. 59-77, El Ateneo, Buenos Aires.

- (1973). Reason and teaching, RKP, Londres.

Sherman, N. (1989). The fabric of character, Aristotle's theory of virtue, Clarendon Press, Oxford.

SCHEUERL, H. (1984). Sobre la cuestión de la fundamentación de las decisiones pedagógicas, Educación (Tubinga), vol. 30, pp. 78-94.

SCHÖN, D. A. (1983). The reflective practitioner, How professionals think in action, Basic Books, Nueva York.

- (1987). Educating the reflective practitioner, Jossey-Bass, San Francisco.

- (1992). The theory of Inquiry: Dewey's legacy to education Curriculum Inquiry, 2:2, pp. 120 139.

SCHWAB, J. (1985). «Un enfoque práctico como lenguaje para el currículum», en J. Gimeno y A. PÉREZ GómEZ (eds.). La enseñanza: su teoría y su práctica, pp. 197-208.

SMITH, J. (ed.) (1987). Educating teachers. Changing the nature of pedagogical knowledge, The Falmer Press, Londres.

- (1986). Reflectiong in action, Deakin University Press, Greelong.

SocketT, H. (1987). Has Schulman got the strategy right?, Harvard Educational Review, 57:2.

- (1989). A moral epistemology of practice?, Cambridge Journal of Education, 19:1, pp. 33-39.

- (1989). «Research, practice and professional aspiration within teaching», Journal of Curriculum Studies, 21:2, pp. 97-112.

Souviron, J. M. (1988). La configuración jurídica de las profesiones tituladas, Consejo de Universidades, Madrid. 
STENHOUSE, L. (1984). Investigación y desarrollo del currículum, Morata, Madrid.

- (1987). La investigación como base de la enseñanza, Morata, Madrid.

Strike, K. A. y Soltis, J. F. (1985). The ethics of teaching, Teachers College Press, Nueva York.

Strike, K. A.; HAller, E. J. y Soltis, J. F. (1988). The ethics of school administration, Teacher College Press, Nueva York.

Sullivan, W. (1986). Reconstructing public philosophy, University of California Press, Berkeley.

TAKATURA, A. (1971). «Practice and production», en Aristotle's theory of practical cognition, pp. 138-164, H. Nijhoff, The Haghe.

TERHART, E. y DreruP, H. (1981). «Knowledge utilization in the science of teaching», British Journal of Educational Studies, 29:1, pp. 9-28.

Thiebault, C. (1988). «En el principio era la acción», en Cabe Aristóteles, pp. 107-142.

TIBBLE, J. W. (comp.). The study of education, RKP, Londres.

ToM, A. (1984). Teaching as a moral craft, Longman, Nueva York.

TOURIÑÁN, J. M. (1984). «Delimitación de la intervención educativa como acción», Revista Española de Pedagogía, XLII:163, pp. 79-97.

- (1987). Teoría de la educación. La educación como objeto de conocimiento, Anaya, Madrid.

- (1987). Formación pedagógica y profesiones de la educación, Bordón, 39:266.

- (1989). «Las finalidades educativas: análisis teórico», en J. M. ESTEVE (ed.). Objetivos y contenidos de la educación para los años noventa, Universidad de Málaga, Málaga.

Trumbull, D. J. (1983). «Practitioner knowledge. An Examination of the artistry in teaching», The Journal of Educational Thought, 20:3, pp. 113-124.

Tvershy, A. y Kahnemann, D. (1974). «Judgement under uncertainty», Science, 185, pp. 11241131.

VICKERS, G. (1983). The art of judgement, Harper and Row, Londres.

VILlA, A. (Coord.). Perspectivas y problemas de la función docente, Narcea, Madrid.

Villar Angulo, L. M. (1990). El profesor como profesional, Servicio de Publicaciones de la Universidad de Granada, Granada.

Villar Angulo, L. M. (dir.) (1988). Conocimiento, creencias y teorías de los profesores, Marfil, Alcoy.

WARNOCK, M. (1989). Una politica común de educación, Paidós Ibérica-MEC, Barcelona.

WeBER, M. (1984). «La naturaleza de la acción social», en La acción social, Ensayos metodológicos, pp. 1-48, Península, Barcelona.

WeItZAKer, C. F. Von (1981). El problema de una ética en el mundo técnico, Universitas, 19:2.

WeKER, R. (1992). The teacher as expert, State University of New York Press, Nueva York.

WestbuRy, I. y WILKOF, N. J. (1978). Science, curriculum and liberal education. Selected essays of J. J. Schwab, University of Chicago Press, Chicago.

WhITE, J. (1976). Teacher accountability and school autonomy. Proceeding of the Philosophy of Education, Society of Great Britain, pp. 58-78.

- (1990). Education and the good life, Kogan Page, Londres. 
WidLAK, H. (1984). El shock que produce la práctica. El fracaso de la aplicación del saber. Educación, Tubinga, vol. 30, pp. 95-107.

WigGER (1984). «Acción y educación. Un análisis crítico de las concepciones de acción en las teorías educativas», Educación, Tubinga, vol. 30, pp. 39-59.

Wittrock, M. (ed.) (1989). La investigación sobre la enseñanza, tres vols., Paidós Ibérica-MEC, Barcelona.

YzQuierdo Tolsada, M. (1989). La responsabilidad civil del profesional liberal, Reus, Madrid.

Zabalza, M. (1987). Pensamiento del profesor y desarrollo didáctico, Enseñanza, $\mathrm{n}^{\circ}$ 4-5, pp. 109-138.

ZARET, E. (1981). «The uncertainty principle in curriculum», Theory into practice, 25:1, pp. 4652. 